Full Paper

\title{
Voltammetric Determination of Meloxicam in Pharmaceutical Formulation and Human Serum at Glassy Carbon Electrode Modified by Cysteic Acid Formed by Electrochemical Oxidation of $L$-cysteine
}

\author{
Cheng Yin Wang ${ }^{1}{ }^{*}$, Zhi Xian Wang ${ }^{1}$, Jun Guan ${ }^{2}$ and Xiao Ya Hu ${ }^{1}$,* \\ ${ }^{1}$ Department of Chemistry and Chemical Engineering, Yangzhou University, Yangzhou 225002, \\ China; E-mail: yzswcy@yzcn.net or wangcy@yzu.edu.cn (Cheng Yin Wang), \\ xiaofeixia1982@163.com (Zhi Xian Wang).xyhu@yzu.edu.cn (Xiao Ya Hu). Phone: 086-0514- \\ 7975590-9217. Fax: 086-0514-7975244 \\ ${ }^{2}$ Subei Hospital, Yangzhou 225002, China; E-mail: Junguan_tt@163.com (Jun Guan) \\ * Authors to whom correspondence should be addressed
}

Received: 5 July 2006 / Accepted: 11 August 2006 / Published: 27 September 2006

\begin{abstract}
The improvement of electrochemical detection of meloxicam is presented by modification of a glassy carbon electrode with anionic layer of cysteic acid providing electrostatic accumulation of the analyte onto the electrode surface. The modification formed by electrochemical oxidation of $L$-cysteine was performed by cycling potential in cysteine solution. The anodic peak current obtained at $+1.088 \mathrm{~V}$ (vs. Ag/AgCl) by voltammetry was linearly dependent on the meloxicam concentration in the range of $4.3 \times$ $10^{-8} \sim 8.5 \times 10^{-6} \mathrm{M}$ in the B-R buffer solution $(0.04 \mathrm{M}, \mathrm{pH} 1.86)$ with a correlation coefficient of 0.999 . The detection limit $(\mathrm{S} / \mathrm{N}=3)$ is $1.5 \times 10^{-9} \mathrm{M}$. The low-cost modified electrode shows good sensitivity, selectivity and stability and has been applied to the determination of meloxicam in pharmaceutical formulation and spiked serum with satisfactory results. The electrochemical reaction mechanism of meloxicam was discussed.
\end{abstract}

Keywords: cysteic acid; L-cysteine; meloxicam; voltammetry; human serum

\section{Introduction}

Meloxicam, (Scheme 1) (4-hydroxy-2-methyl-N-(5-methyl-2-thiazoly)-2H-1,2-benzo-thia- zine-3carbox-amide-1,1dioxide) $\left(\mathrm{C}_{14} \mathrm{H}_{13} \mathrm{~N}_{3} \mathrm{O}_{4} \mathrm{~S}_{2}\right)$ is a novel nonsteroidal anti-inflammatory drug (NSAID) with a favorable COX-2 (cyclooxgenase-2) [1,2]: COX-1 (cyclooxgenase-1) selectivity has also been 
shown to have potent anti-inflammatory effects [3,4]. Because of solubility of meloxicam in acidic medium, it may cause local gastrointestinal adverse events [5]. Up to now, there are a limited number of techniques described for the determination of meloxicam in pharmaceuticals formulations only including ultraviolet spectrophotometry [6], flow injection [7] and high-performance liquid chromatography (HPLC) with UV detector [8,9]. The Chinese pharmacopoeia method for conventional assaying meloxicam is based on neutralization titration in the solvent of ethanol [10]. However, There have been a very few methods have been published about the determination of meloxicam in biological media only including capillary zone electrophoresis (CZE) [11] and HPLC [12]. Further more, many of methods mentioned above required time-consuming manipulation steps, sophisticated instruments and special training. For these reasons, the rapid, simple and accurate method for the determination with high sensitivity is expected to be established.

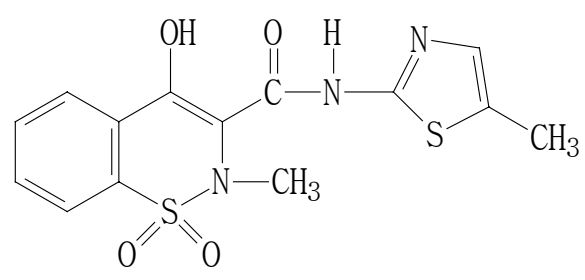

Scheme 1. Structure of meloxicam.

Electrochemical detection of analytes is a very elegant method in analytical chemistry. The interest in developing electrochemical sensing devices for use in environmental monitoring, clinical assays or process control is growing rapidly. Electrochemical sensors satisfy many of the requirements for such tasks particularly owing to their inherent specificity, speed of response, sensitivity and simplicity of preparation. Up to date, there are only three available electrochemical methods for the determination in the literature including polarography with the mercury electrode [13,14] and voltammetry [15]. However, the polarography would contaminate the environment for the mercury electrode if the mercury is handled with no special care. Radi et al. proposed a voltammetric method with a carbon paste electrode for the determination of meloxicam [15], and the linear range was $5.0 \times 10^{-7} \sim 5.0 \times 10^{-}$ ${ }^{5} \mathrm{M}$ with the detection limit of $1.6 \times 10^{-7} \mathrm{M}$. These electrochemical methods were only described for the determination of meloxicam in pharmaceutical formulations. There is no literature reported about quantitative determinations of meloxicam in biological media by any electrochemical method.

In the present paper we described a rapid, simple voltammetric method to detect meloxicam in human serum with a glassy carbon electrode modified by cysteic acid formed by electrochemical oxidation of $L$-cysteine. A sensitive anodic oxidative peak of meloxicam was used for the quantitative determination. A good linear relationship was realized between the anodic peak currents and meloxicam concentrations in the range of $4.3 \times 10^{-8} \sim 8.5 \times 10^{-6} \mathrm{M}$ with the detection limit of $1.5 \times 10^{-}$ ${ }^{9} \mathrm{M}$. By comparison of the results obtained at bare glassy carbon electrode and carbon paste electrode [15], the modification provided higher signal toward meloxicam in a broad range of experimental conditions. The performance of the modified electrode was illustrated by determination of meloxicam in pharmaceutical preparations and spiked serum. This method has the advantages of lower 
interference in the serum, rapid and simple operation, and high accuracy. This film is considered to be a promising, low-cost, steady and biocompatible material of electrode modifiers.

\section{Experimental Section}

\subsection{Apparatus}

Voltammetry experiments were carried out at a CHI 660B electrochemical workstation (Chenhua Instruments, China). All electrochemical experiments employed a conventional three-electrode system with a glassy carbon electrode or a cysteic acid modified glassy carbon electrode ( $4.0 \mathrm{~mm}$ in diameter) as a working electrode, a platinum wire as an auxiliary electrode and an $\mathrm{Ag} / \mathrm{AgCl}$ electrode as a reference electrode. All potentials reported in this paper were referenced to the Ag/AgCl electrode. All experiments were carried out at $25^{\circ} \mathrm{C}$.

\subsection{Chemicals and Solutions}

L-cysteine (CySH) was obtained from Shanghai Chemical Reagent Co., Ltd. (Shanghai, China). Meloxicam was purchased from Sigma (Milwaukee, Wisconsin, USA). Nafion (5 wt \% in alcohols) was purchased from Fluka (Milwaukee, Wisconsin, USA). All the other reagents used were of analytical grade. Doubly distilled water was obtained by purification through a Millipore water system and used throughout. A stock anhydrous ethanol solution of meloxicam $\left(1.0 \times 10^{-3} \mathrm{M}\right)$ was prepared, and kept in the dark under refrigeration (below $4{ }^{\circ} \mathrm{C}$ ). Britton-Robinson (B-R) buffer solutions at various $\mathrm{pH}$ values were used as base solutions for the determination of meloxicam.

\subsection{Fabrication of the Cysteic Acid Modified Glassy Carbon Electrodes}

Before modification, the glassy carbon electrode was polished with $1,0.3$ and $0.05 \mu$ m $\alpha$-alumina powder, respectively, rinsed thoroughly with doubly distilled water within each polishing step. And then it was sonicated successively in 1:1 nitric acid, acetone and doubly distilled water. The modified electrode was prepared by cycling the potential between -1.2 and $+2.6 \mathrm{~V}$ at the scan rate of 100 $\mathrm{mV} \cdot \mathrm{s}^{-1}$ in $0.04 \mathrm{M} \mathrm{HCl}$ solution containing $2.5 \times 10^{-3} \mathrm{M} \mathrm{L}$-cysteine. Fig. 1 shows the cyclic voltammograms of $L$-cysteine at a bare glassy carbon electrode. After 10 consecutive cycles, the surface of the glassy carbon electrode was covered with certain blue substance observed. Then, the modified electrode was rinsed respectively with ethanol and distilled water to remove the physically adsorbed and unreacted species from the electrode surface. Subsequently, the modified electrode was electroactivated by cyclic scanning from -0.2 to $+1.2 \mathrm{~V}$ in the B-R buffer solution (0.04 M, pH 1.86), and a steady cyclic voltammogram was obtained. Finally, the electrode was dried with a stream of high purity nitrogen.

\subsection{Determination of Meloxicam}

The cysteic acid modified glassy carbon electrode, the platinum wire counter electrode and the $\mathrm{Ag} / \mathrm{AgCl}$ reference electrode were immersed in $20.0 \mathrm{~mL}$ the B-R buffer solution (0.04 M, pH 1.86). A certain amount of meloxicam was added into the solution being stirred by a magnetic stirrer. The 
agitation was stopped after the electrochemical accumulation for $300 \mathrm{~s}$ at $-0.2 \mathrm{~V}$. Then the voltammetry was immediately performed to scan from 0.4 to $1.4 \mathrm{~V}$ after $30 \mathrm{~s}$ of quiet time. The two anodic peaks currents of meloxicam at $+0.695 \mathrm{~V}(\mathrm{p} 1)$ and $+1.088 \mathrm{~V}(\mathrm{p} 2)$ were recorded (Fig. 2). The standard addition method was applied for the quantitative determination of meloxicam at $+1.088 \mathrm{~V}$ (p2). After the determination, the renewal of the electrode was accomplished by soaking it in the B-R buffer solution and cycling potentials between -0.2 and $1.2 \mathrm{~V}$ about 6 cycles when there was no peak.

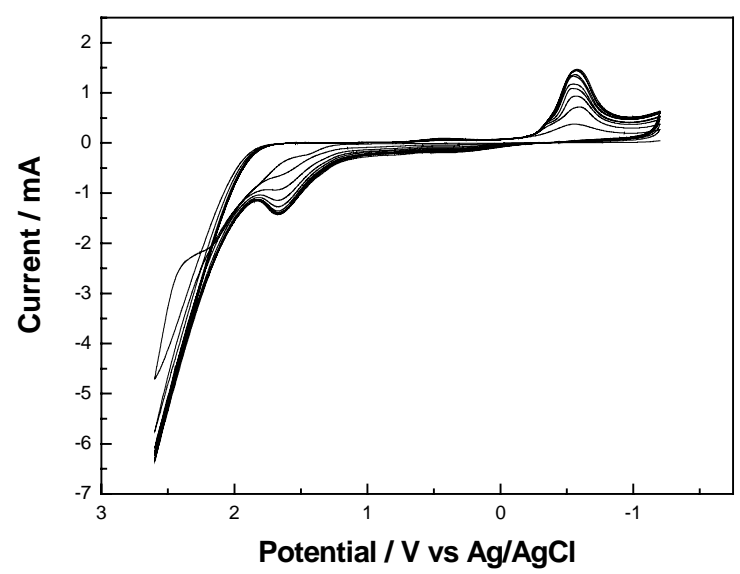

Figure 1. Cyclic voltammograms of $2.5 \times 10^{-3} \mathrm{M} \mathrm{L}$-cysteine at a glassy carbon electrode in $0.04 \mathrm{M}$ $\mathrm{HCl}$ solution. Scan potential: -1.2 to $2.6 \mathrm{~V}$; scan rate: $100 \mathrm{mV} \cdot \mathrm{s}^{-1}$.

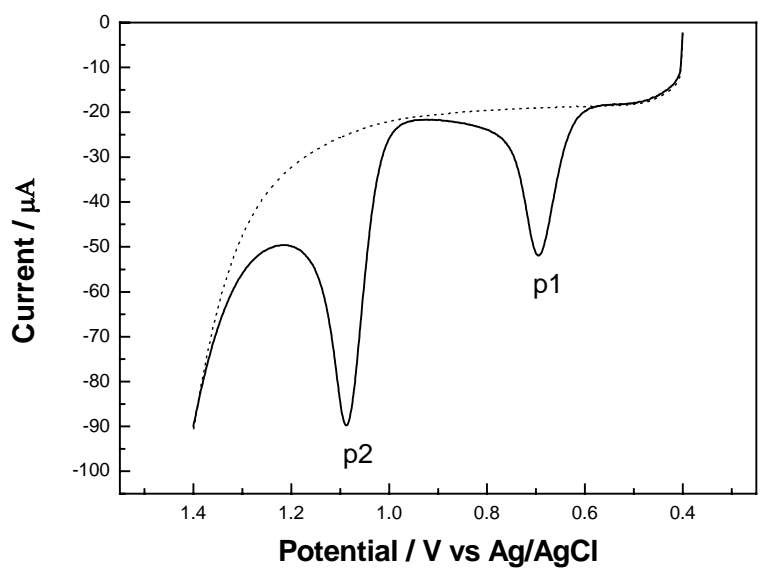

Figure 2. Adsorptive voltammograms of $3.5 \times 10^{-6} \mathrm{M}$ meloxicam (solid line) and blank (dot line) at the modified electrode in the B-R buffer solution (0.04 M, pH 1.86). Accumulation potential under stirring: - $0.2 \mathrm{~V}$; accumulation time: $300 \mathrm{~s}$; quiet time: $30 \mathrm{~s}$; scan rate: $100 \mathrm{mV} \cdot \mathrm{s}^{-1}$.

\subsection{Coulometry Experiments}

The coulometry was carried out to determine number of electrons involved in the oxidation process of meloxicam. The meloxicam solution $\left(2.5 \times 10^{-6} \mathrm{M}\right)$ was electrolyzed exhaustively at $+1.088 \mathrm{~V}(\mathrm{p} 2)$ and $+0.695 \mathrm{~V}(\mathrm{p} 1)$ respectively until the electrolytic currents were close to zero, and the quantity of 
electrical charge $Q_{1}$ was recorded. Likewise, the background solution was electrolyzed under the same conditions, and the quantity of electrical charge $Q_{2}$ was obtained.

\section{Results and Discussion}

\subsection{The Role of Materials Modified on the Glassy Carbon Electrode}

Many electrochemical researchers have been devoted to determination of CySH [16,17]. And it is widely accepted that the reaction occurred by the following oxidation reaction mechanism on electrodes [17-20]:

$$
\begin{gathered}
\mathrm{CySH} \leftrightarrow \mathrm{CyS}^{-}+\mathrm{H}^{+} \\
\mathrm{CyS}^{-} \rightarrow \mathrm{CyS}_{\text {ads }}^{\cdot}+\mathrm{e}^{-} \\
2 \mathrm{CyS}_{\text {ads }} \rightarrow \mathrm{CySSCy}
\end{gathered}
$$

Herein, electrochemical oxidation of $\mathrm{CySH}$ at a glassy carbon electrode was also investigated by the cyclic voltammetry in the wide potential range of $-1.2 \sim 2.6 \mathrm{~V}$ at a scan rate of $100 \mathrm{mV} \cdot \mathrm{s}^{-1}$ in $0.04 \mathrm{M}$ $\mathrm{HCl}$ solution containing $2.5 \times 10^{-3} \mathrm{M} \mathrm{CySH}$. Fig. 1 shows the cyclic voltammograms of $L$-cysteine at a glassy carbon electrode. There was an irreversible oxidation peak at ca. $+0.5 \mathrm{~V}$ at the first segment, which implied that CySH was oxidized to L-cystine (CySSCy). And another irreversible oxidation peak was observed at higher potential (ca. $+1.6 \mathrm{~V}$ ) with further voltammetric cycling. In many cases, cysteic acid $\left(\mathrm{CySO}_{3} \mathrm{H}\right)$ was found as an oxidation product of CySSCy [18-21]. Fei et al. described that the oxidation product of $\mathrm{CySH}$ can be further oxidized to chemisorbing molecules (cysteic acid) under high positive potential [20]. Ralph et al. demonstrated that $\mathrm{CySH}$ was adsorbed on the surface of a glassy carbon electrode by using AC voltammetry, and its further oxidation to form cysteic acid was proposed [19]:

$$
\mathrm{RSSR}+3 \mathrm{H}_{2} \mathrm{O} \rightarrow \mathrm{RSO}_{3} \mathrm{H}+5 \mathrm{H}^{+}+5 \mathrm{e}^{-}
$$

Spataru et al. confirmed that the functional group $\mathrm{SO}_{3} \mathrm{H}$ of cysteic acid was strongly adsorbed at a glassy carbon electrode by using cyclic voltammetric and polarization measurements [17]. According to its chemical structure, cysteic acid formed by the electrochemical oxidation of $\mathrm{CySH}$ is similar to Nafion with sulfonated groups, so it can be used as a novel electrode modifier due to its attractive ionexchange characteristics. Fig. 6 shows the peak current response of meloxicam at cysteic acid/GCE is enhanced compared with the bare GCE under above identical experimental conditions. It was attributed to the electrostatic attraction of modifier layer for the amine groups of meloxicam molecules carrying positive charges in the acid solution. In order to confirm this property, Nafion film which was characteristic of cation exchange was coated on the surface of the GCE to prepare Nafion/GCE for comparison with the proposed modifier material. $0.1 \mathrm{wt} \%$ Nafion solution used in this work was prepared by diluting the $5 \mathrm{wt} \%$ Nafion solution in ethanol. A same GCE was coated with $10 \mu \mathrm{L}$ of the Nafion solution above mentioned. The solvent was left to evaporate at room temperature in air. The two different modified electrodes all improved current responses of meloxicam compared with bare GCE under above identical experimental conditions, implying that cysteic acid and Nafion played the 
same role in the accumulations of meloxicam. Fig. 3 shows that the oxidation peak potentials at the Nafion/GCE are similar to those at the cysteic acid/GCE. But the current response at the Nafion/GCE is lower than that at cysteic acid/GCE, and it decreases one order of magnitude compared with the cysteic acid/GCE because of poor conductivity of Nafion. Additionally, Nafion is more expensive than $\mathrm{CySH}$. So cysteic acid film has more significant advantages and electroanalytical application than Nafion film. On the other hand, we found that the modified electrode itself had $\mathrm{pH}$ sense under different $\mathrm{pH}$ environments and exhibited a good $\mathrm{pH}$ linear region from 2.0 to 12.0 with a nearNernstian slope by experiment. The cation exchange property of the modifier was confirmed further. Consequently, a novel solid $\mathrm{pH}$ sensor will be fabricated using the thin membrane materials of cysteic acid supported on a glassy carbon electrode in our subsequence research.
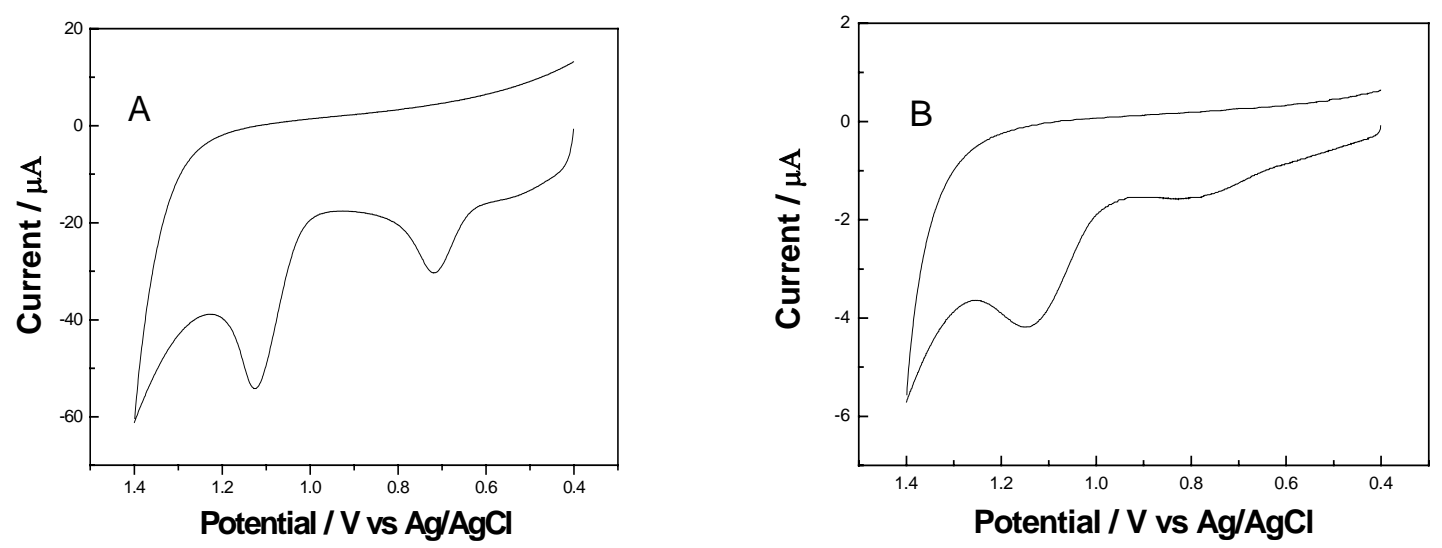

Figure 3. Cyclic voltammograms of $5.0 \times 10^{-7} \mathrm{M}$ meloxicam at different modified GCEs. (A) cysteic acid/GCE; (B) Nafion/GCE. Scan rate: $100 \mathrm{mV} \cdot \mathrm{s}^{-1}$; accumulation potential under stirring: - $0.2 \mathrm{~V}$; accumulation time: $300 \mathrm{~s}$; quiet time: $30 \mathrm{~s}$; electrolyte: B-R buffer solution (0.04 M, pH 1.86).

\subsection{Optimization of Experimental Conditions}

Some conventional supporting electrolytes were tested, such as $\mathrm{HCl}, \mathrm{H}_{2} \mathrm{SO}_{4}$, HAc-NaAc, $\mathrm{NH}_{4} \mathrm{Cl}$ $\mathrm{NH}_{3} \cdot \mathrm{H}_{2} \mathrm{O}, \mathrm{KCl}$, phosphate buffer and B-R buffer solution. But higher sensitive and well-defined peaks of meloxicam in voltammograms were obtained in the B-R buffer solution (0.04 M, pH 1.86). The effects of accumulation potential and accumulation time on the voltammetric current response for meloxicam were studied. The accumulation step proceeded in constantly stirred solution and the voltage-scanning step was performed after $30 \mathrm{~s}$ of quiet time. The peak current of meloxicam was the highest at $-0.2 \mathrm{~V}$ as the accumulation potential. It is attributed to the existence of meloxicam in the cationic form at $\mathrm{pH}$ 1.86, and more favorable accumulation at negative potential. So an accumulation potential of - $0.2 \mathrm{~V}$ was chosen in all the subsequent work. The effect of accumulation time on the peak current was investigated, too. Fig. 4 shows the peak currents of meloxicam increased with the increasing the accumulation time within $300 \mathrm{~s}$, which is attributed to the adsorption of meloxicam at the modified electrode surface. Further postponement of the accumulation time did not increase the response of meloxicam after $300 \mathrm{~s}$ owing to the surface adsorption saturation. For practical purposes, a $300 \mathrm{~s}$ accumulation period was found to be sufficient for the determination of meloxicam. 


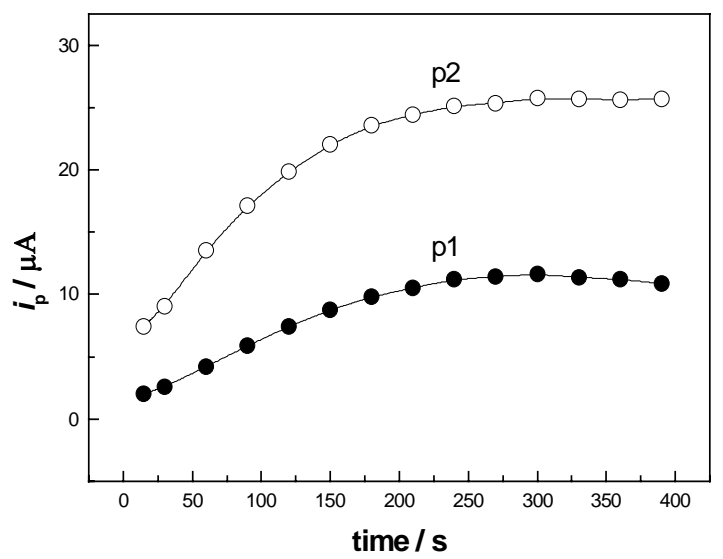

Figure 4. Effect of the accumulation time on the anodic peak currents of $5.0 \times 10^{-7} \mathrm{M}$ meloxicam in the B-R buffer solution (0.04 M, pH 1.86). Scan rate: $100 \mathrm{mV} \cdot \mathrm{s}^{-1}$; accumulation potential: $-0.2 \mathrm{~V}$; quiet time: $30 \mathrm{~s}$.

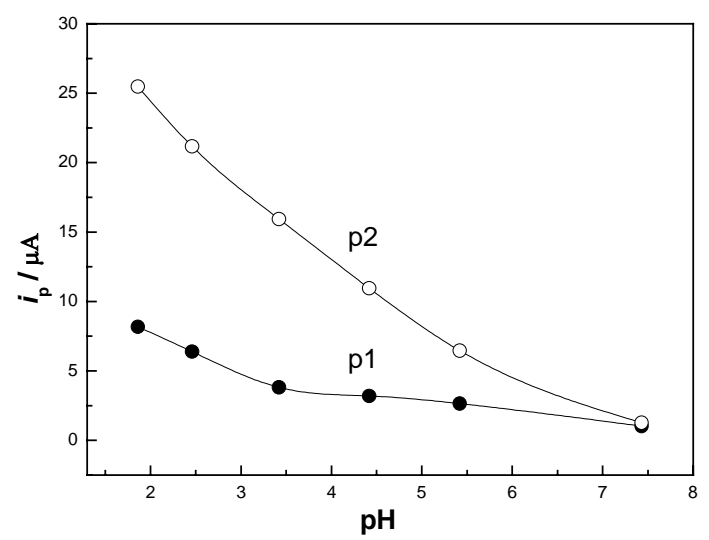

Figure 5. Effect of $\mathrm{pH}$ on the anodic peak currents (p1, p2) of $5.0 \times 10^{-7} \mathrm{M}$ meloxicam at the cysteic acid/GCE. Scan rate: $100 \mathrm{mV} \cdot \mathrm{s}^{-1}$; accumulation potential: $-0.2 \mathrm{~V}$; accumulation time: $300 \mathrm{~s}$; quiet time: 30 s; electrolyte: B-R buffer solution (0.04 M).

The $\mathrm{pH}$ value of the base solution has a significant influence on oxidation current response of meloxicam at the cysteic acid/GCE. Fig. 5 shows the effect of $\mathrm{pH}$ value of the base solution in the range of $1.86 \sim 7.43$ on the peak currents (p1 and p2). The peak currents increased monotonically with $\mathrm{pH}$ value decreasing. But the interference of water electrolysis became serious when the $\mathrm{pH}$ value was lower than 1.86. So the B-R buffer solution (0.04 M, pH 1.86) was selected for the determination.

The anodic peak currents of meloxicam were considerably related to the amount of cysteic acid on the surface of the glassy carbon electrode. When the electrode was carried out the sweeps under the cyclic voltammetry, the more cycles of sweep, the more cysteic acid was oxidized on the surface of the electrode. Fig. 6 shows the remarkably effect of cysteic acid contents on the peak currents of meloxicam. The anodic peak currents at the cysteic acid/GCE are obviously higher than that on the bare glassy carbon electrode. It might be ascribed to the accumulation of cysteic acid for meloxicam. The anodic peak currents gradually increased with increasing the amount of cysteic acid on the surface 
of the electrode. However, the anodic peak currents decreased when excessive cysteic acid was oxidized at the electrode. We owed it to the decline of the electrode conductivity. So a 10-circle-sweep by the cyclic voltammetry was selected in present experiments.

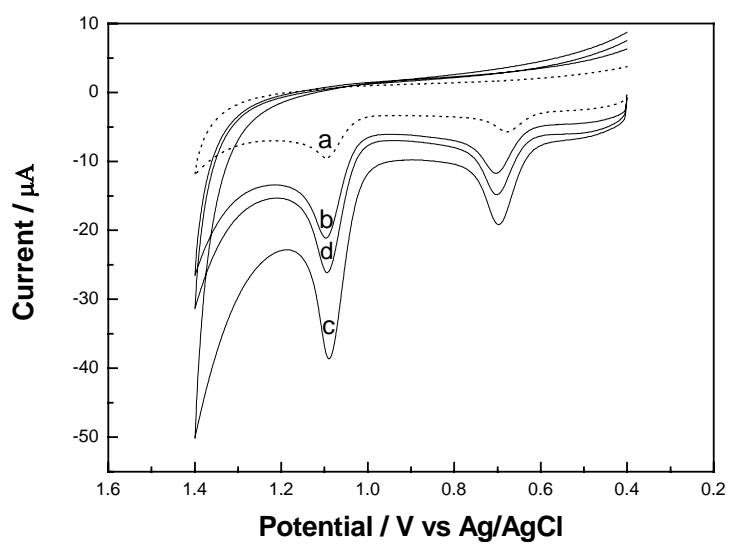

Figure 6. Cyclic voltammograms of $3.5 \times 10^{-6} \mathrm{M}$ meloxicam at the cysteic acid/GCEs prepared in modified solution with different sweep circles. a, the bare glassy carbon electrode; b, 5 circles; c, 10 circles; d, 15 circles. Scan rate: $100 \mathrm{mV} \cdot \mathrm{s}^{-1}$; electrolyte: B-R buffer solution (0.04 M, pH 1.86).

\subsection{Electrochemical Reaction Mechanism of Meloxicam at the Cysteic Acid Modified Electrode}

The coulometry was carried out to determine number of electrons involved in the oxidation of meloxicam. The meloxicam solution $\left(2.5 \times 10^{-6} \mathrm{M}\right)$ was electrolyzed exhaustively at $+1.088 \mathrm{~V}(\mathrm{p} 2)$ and $+0.695 \mathrm{~V}$ (p1) respectively until the electrolytic currents were close to zero, and the quantity of electrical charge $Q_{1}$ was recorded. Likewise, the background solution was also electrolyzed under the same conditions, and the quantity of electrical charge $Q_{2}$ was obtained.

The charge difference $Q=\left(Q_{1}-Q_{2}\right)$ represents the quantities of electrical charge consumed by oxidation of the meloxicam:

$$
Q=n F C V
$$

Here, $C$ and $V$ here are the concentration and volume of the meloxicam solution, respectively. $n$ and $F$ has their conventional meanings. From $E_{q}$. (5), the electron numbers both $n(\mathrm{p} 2)$ and $n(\mathrm{p} 1)$ of the electrochemical reaction of meloxicam could be approximately calculated to be 1 , respectively. The experimental results indicated that there was a good linear relationship between the two peaks' potentials and $\log v$ with the slope of 0.1389 and 0.1014 which equals $0.059 / \alpha n$ according to the Nernst equation. Here $\alpha$ is the coefficient of electrons transfer. Then both $\alpha n$ (p2) and $\alpha n$ (p1) is found to be 0.43 and 0.58 , respectively. The $\mathrm{pH}$ dependence on the peak potentials was also investigated, and they were observed to shift towards positive direction with decrease of $\mathrm{pH}$ value. According to our results from experiments, we found that the modified electrode itself had $\mathrm{pH}$ sense under different $\mathrm{pH}$ environments and exhibited a good $\mathrm{pH}$ linear region from 2.0 to 12.0 with a near-Nernstian slope. But, under different $\mathrm{pH}$ environments, we wanted to know if the charge of cysteic acid layer on the electrode surface influenced the peak potential of meloxicam in Faradic process with the transfer of the hydrogen ion. A voltammetry experiment was performed at the modified electrode using KI as a redox probe $\left(\mathrm{I}_{2}+2 \mathrm{e}=2 \mathrm{I}^{-}\right)$without any transfer of the hydrogen ion in its Faradic process. We found the 
anodic peak of the iodide was constant in variable $\mathrm{pH}$ solution ranged from 2 to 8 . So the $\mathrm{pH}$ dependence on the peak potential of meloxicam reflected only the transfer of the hydrogen ion in its Faradic process. A good linear relationship was obtained between the peak potential (p2) and $\mathrm{pH}$ value in the range of $\mathrm{pH} 1.86$ to 7.43 with the slope of -0.0987 from Nernst equation $E_{p}=K-(0.059 \mathrm{~m} / \mathrm{om})$. $\mathrm{pH}$. Here $m$ was the number of $\mathrm{H}^{+}$transfer. The $m$ could be calculated to be 0.89 . So $m$ was 1 approximately. From above discussion, the oxidation process of the meloxicam involves two electrons and one proton. The result is consistent with the reference [15].

The scan rate effect on the peak current of $5.0 \times 10^{-7} \mathrm{M}$ meloxicam at the modified electrode was also investigated. It could be seen from Fig.7 that the anodic peak current and cathodic peak current increased with increasing the scan rate. Two good linearity between $i_{p}$ and the scan rate $v$ were obtained from the range of $5 \sim 150 \mathrm{mV} \cdot \mathrm{s}^{-1}$, which demonstrated that the electrode reaction was an adsorption-controlled process [22].

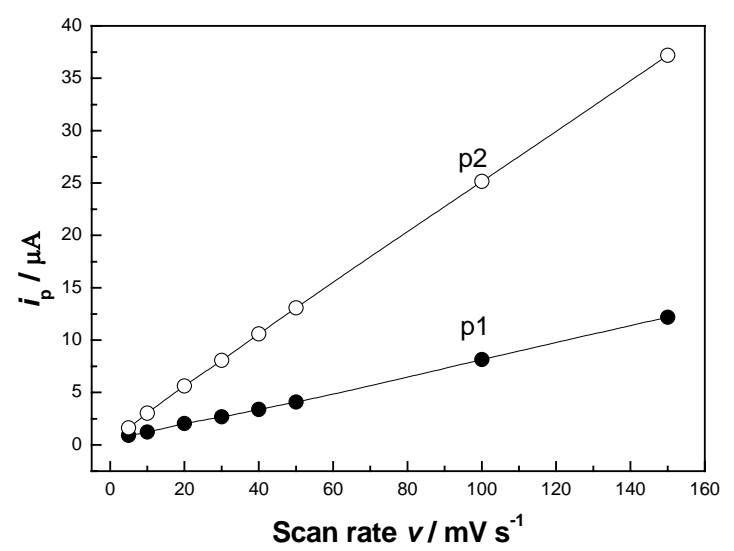

Figure 7. Plots of the anodic peak currents of $5.0 \times 10^{-7} \mathrm{M}$ meloxicam at the cysteic acid/GCE in the B-R buffer solution $(0.04 \mathrm{M}, \mathrm{pH} 1.86)$ at different scan rates. $\mathrm{p} 1$, the peak current at $+0.695 \mathrm{~V}$; $\mathrm{p}$, the peak current at $+1.088 \mathrm{~V}$. Accumulation potential: $-0.2 \mathrm{~V}$; accumulation time: $300 \mathrm{~s}$; quiet time: $30 \mathrm{~s}$.

\subsection{Calibration Curve and Detection Limit}

Under above optimum detection conditions, the anodic peak currents at $+1.088 \mathrm{~V}(\mathrm{p} 2)$ recorded were proportional to meloxicam concentration in the range of $4.3 \times 10^{-8} \sim 8.5 \times 10^{-6} \mathrm{M}$. The linear equation is $i_{\mathrm{pa}}(\mu \mathrm{A})=21.5+8.4 C\left(10^{-6} \mathrm{M}\right)$ at $+1.088 \mathrm{~V}$ with the correlation coefficient $\mathrm{r}=0.999$. The detection limit of meloxicam was $1.5 \times 10^{-9} \mathrm{M}$ in terms of signal to noise ratio of 3:1 $(\mathrm{S} / \mathrm{N}=3)$. When the concentration of meloxicam was more than $8.5 \times 10^{-6} \mathrm{M}$, the current response decreased gradually and its plateau was observed, it was attributed to the saturation of meloxicam at the modified electrode.

\subsection{Repeatability}

The lifetime of the cysteic acid/GCE was examined, and the result demonstrated that the cysteic acid film retained $98.8 \%$ of its initial response after 1 month of storage in B-R buffer solution $(0.04 \mathrm{M}, \mathrm{pH}$ 1.86). After detecting the meloxicam in every five serum samples, the electrode was washed in the B$\mathrm{R}$ buffer solution under stirring, and then transferred into a standard solution of $4.5 \times 10^{-6} \mathrm{M}$ 
meloxicam to record its oxidation peak current (p2). Its average current was $57.7 \mu \mathrm{A}$ with the relative standard deviation (RSD) of $2.2 \%(n=5)$. Such stability seemed to be acceptable for most practical applications. Because the modified electrode could adsorb meloxicam, it was necessary to renew the electrode surface. It was found that the renewal of the electrode surface was easily accomplished by immerging the modified electrode in B-R buffer solution by cycling its potential between - 0.2 and + $1.2 \mathrm{~V}$ about 6 cycles. A quality control chart (X-chart) has been constructed. Fig. 8 shows a quality control chart (X-chart) of $4.5 \times 10^{-6} \mathrm{M}$ meloxicam applying the proposed method, where the $\mathrm{X}$-axis represents the number of analyses while the Y-axis represents the anodic current (p2) of meloxicam. There is a series of 15 points over the average score and all of them fall in between the upper control limit (UCL) and the lower control limit (LCL), which indicated that the analysis progress was under the statistical control.

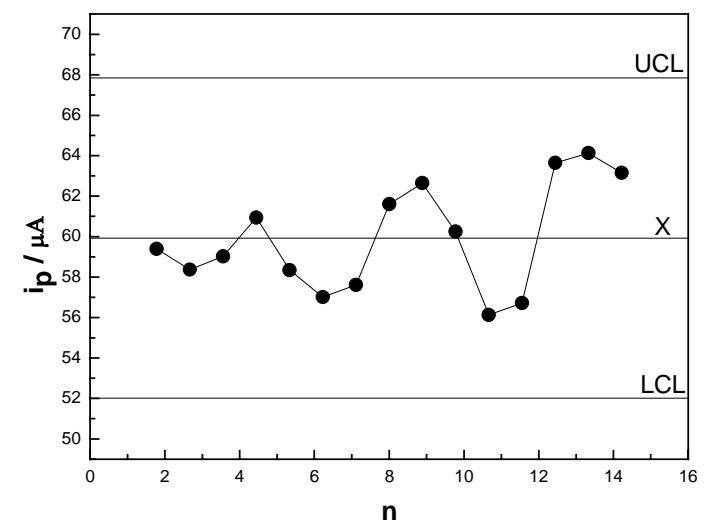

Figure 8. Quality control chart (X - Chart) of $4.5 \times 10^{-6} \mathrm{M}$ meloxicam.

\subsection{Recovery Test}

The recovery tests of meloxicam in the range from $2.8 \times 10^{-7}$ to $1.06 \times 10^{-6} \mathrm{M}$ were performed. The results are listed in Table 1. The recoveries varied in the range from $96.4 \%$ to $102.6 \%$ and the RSD was $2.4 \%$.

Table 1. Recovery of meloxicam.

\begin{tabular}{rrc}
\hline Added / M & Found / M & Recovery (\%) \\
\hline $2.8 \times 10^{-7}$ & $2.7 \times 10^{-7}$ & 96.4 \\
$3.8 \times 10^{-7}$ & $3.9 \times 10^{-7}$ & 102.6 \\
$7.6 \times 10^{-7}$ & $7.7 \times 10^{-7}$ & 101.3 \\
$8.6 \times 10^{-7}$ & $8.5 \times 10^{-7}$ & 98.8 \\
$1.06 \times 10^{-6}$ & $1.05 \times 10^{-6}$ & 99.1 \\
\hline
\end{tabular}

\subsection{Interference}

For the following solid pharmaceutical formulations analysis, tablet excipient, such as starch, was added in the detection system. It was not found that the results were changed in the presence of the excipient when $5.0 \times 10^{-7} \mathrm{M}$ meloxicam was detected under the optimum experimental conditions. If the anodic current of the first oxidation potential (p1) was selected for the determination in serum 
samples referred to the previous literature [15], uric acid (UA) and ascorbic acid (AA) in existence would severely interfere with the determination of the meloxicam because of the overlapping voltammetric response of UA and AA. Whereas the presence of UA and AA in high concentration did not interfere with the determination of meloxicam when the anodic current of the second oxidation potential $(+1.088 \mathrm{~V})$ was selected for the determination in serum samples. The other conceivable concomitant in serum samples were investigated in this detection system. When the concentration of the meloxicam was $5.0 \times 10^{-7} \mathrm{M}$, no interferences were observed in the presence of $5.0 \times 10^{-4} \mathrm{M}$ of $\mathrm{NaCl}, \mathrm{KNO}_{3},\left(\mathrm{NH}_{4}\right)_{2} \mathrm{SO}_{4}, \mathrm{Mg}\left(\mathrm{NO}_{3}\right)_{2}, \mathrm{Ca}\left(\mathrm{NO}_{3}\right)_{2}$, glucose, oxalic acid, urea, tartaric acid, caffeine, $5.0 \times$ $10^{-5} \mathrm{M}$ of cysteine, cystine, DL-tyrosine, citrate, $\mathrm{CuSO}_{4}, 2.0 \times 10^{-4} \mathrm{M}$ of albumin fraction and $\mathrm{Fe}\left(\mathrm{NO}_{3}\right)_{3}$, respectively. The results obtained are listed in Table 2 . In addition, some conventional medicaments, such as $1.0 \times 10^{-4} \mathrm{M}$ of Vitamin $\mathrm{B}_{1}$, Vitamin $\mathrm{B}_{2}$, Vitamin $\mathrm{B}_{6}$, atropine, penicillin, and theine, did not interfere with the determination.

Table 2. Influence of potential interferents on the voltammetric response of $0.50 \mu \mathrm{M}$ meloxicam.

\begin{tabular}{|c|c|c|}
\hline Interferent & Concentration / mM & $\begin{array}{c}\text { Signal change } \\
\left(i_{\text {meloxicam, } 22}=100 \%\right)\end{array}$ \\
\hline AA & 0.05 & $-0.9 \%$ \\
\hline AA & 0.10 & $-1.8 \%$ \\
\hline AA & 0.20 & $-3.5 \%$ \\
\hline UA & 0.50 & $-2.6 \%$ \\
\hline albumin fraction & 0.20 & $+1.7 \%$ \\
\hline urea & 0.50 & $-2.2 \%$ \\
\hline tartaric acid & 0.50 & $-3.0 \%$ \\
\hline cystine & 0.05 & $-1.9 \%$ \\
\hline citrate & 0.05 & $-2.5 \%$ \\
\hline glucose & 0.50 & $+0.5 \%$ \\
\hline cysteine & 0.05 & $-2.9 \%$ \\
\hline$D L$-tyrosine & 0.05 & $-2.3 \%$ \\
\hline oxalic acid & 0.50 & $-1.9 \%$ \\
\hline caffeine & 0.50 & $+1.5 \%$ \\
\hline $\mathrm{NaCl}$ & 0.50 & $-1.3 \%$ \\
\hline $\mathrm{KNO}_{3}$ & 0.50 & $-2.2 \%$ \\
\hline $\mathrm{Ca}\left(\mathrm{NO}_{3}\right)_{2}$ & 0.50 & $-1.9 \%$ \\
\hline$\left(\mathrm{NH}_{4}\right)_{2} \mathrm{SO}_{4}$ & 0.50 & $-2.4 \%$ \\
\hline $\mathrm{Mg}\left(\mathrm{NO}_{3}\right)_{2}$ & 0.50 & $-1.0 \%$ \\
\hline $\mathrm{CuSO}_{4}$ & 0.05 & $-3.2 \%$ \\
\hline $\mathrm{Fe}\left(\mathrm{NO}_{3}\right)_{3}$ & 0.20 & $-2.6 \%$ \\
\hline
\end{tabular}

\subsection{Determination of the Pharmaceutical Preparation Samples}

The developed voltammetry method for the meloxicam determination was applied to a commercial preparations (meloxicam tablets: Yangzijiang Pharmaceutical Co. Ld., China, Hongqiang ${ }^{\circledR}$, batch No. 04110402, labeled amount of $7.5 \mathrm{mg}$ per tablet). A known number of tablets were grounded to fine powers, and an accurate mass of the powers about $200.0 \mathrm{mg}$ was transferred to a $50.0 \mathrm{ml}$ calibrated flasks. It was extracted for $5 \mathrm{~min}$ with $50.0 \mathrm{ml}$ cold ethanol. Table 3 shows the results of voltammetry 
analysis of the commercial preparations. The Chinese pharmacopoeia methods (acid-base titration) [10] was employed as a comparison to evaluate the validity of the developed method ( $0.4 \mathrm{~g}$ of tablets was dissolved in a mixture of $100 \mathrm{ml}$ of ethanol and acid-base titration was carried out with bromophenol blue as an indicator. Each $1 \mathrm{ml}$ of $0.1 \mathrm{M}$ sodium hydroxide is equivalent to $35.14 \mathrm{mg}$ of meloxicam). A good agreement was achieved and there was no significant difference between methods. The results obtained show the developed method could be easily used in quality control laboratory for the analysis of meloxicam in solid pharmaceutical formulations.

Table 3. Determination results of meloxicam in tablets.

\begin{tabular}{cccc}
\hline Batch No. & $\begin{array}{c}\text { Pharmacopoeial Method } \\
(\mathrm{n}=5)(\mathrm{mg} / \mathrm{tablet})\end{array}$ & $\begin{array}{c}\text { Present method } \\
(\mathrm{n}=5)(\mathrm{mg} / \text { tablet })\end{array}$ & $\begin{array}{c}\text { R.S.D* } \\
(\%)\end{array}$ \\
\hline Hongqiang ${ }^{\circledR} 04110402$ & 7.3 & 7.5 & 2.2 \\
\hline
\end{tabular}

* Here R.S.D is relative standard deviation.

\subsection{Detection of Meloxicam in Human Serum Samples}

The developed voltammetry method for the meloxicam determination was applied to human serum samples. The serum samples were obtained from volunteers. The recovery from human serum was measured by spiking drug-free human serum with known amounts. The serum samples without further pretreatments were diluted 100 times with the 0.04 M B-R buffer solution ( $\mathrm{pH} \mathrm{1.86)} \mathrm{before} \mathrm{analysis.}$ The peak current of meloxicam at $+1.088 \mathrm{~V}$ (p2) were recorded. Standard addition method was employed under above optimal experimental conditions. The detection results obtained are listed in Table 4. The recovery determined was ranged from 96.8 to $103.2 \%$.

Table 4. Determination of meloxicam in spiked serum samples at the cysteic acid/GCE.

\begin{tabular}{cccc|}
\hline Serum & Spiked / $\mu \mathrm{g}$ & Detected* / $\mu \mathrm{g}$ & Recovery / \% \\
\hline Sample 1 & 6.0 & $5.8 \pm 0.2$ & 96.8 \\
Sample 2 & 7.5 & $7.7 \pm 0.3$ & 102.3 \\
Sample 3 & 10.6 & $10.9 \pm 0.4$ & 103.2 \\
Sample 4 & 13.5 & $13.6 \pm 0.4$ & 100.7 \\
Sample 5 & 15.0 & $14.8 \pm 0.5$ & 98.7 \\
Sample 6 & 16.5 & $16.0 \pm 0.6$ & 97.0 \\
\hline
\end{tabular}

* Number of sample assayed was five.

\section{Conclusions}

A novel modified electrode was fabricated using cysteic acid formed by electrochemical oxidation of $L$-cysteine at a glassy carbon electrode. Cysteic acid with cation exchange property drastically increased the current response of meloxicam into two well-defined peaks. The peak current at +1.088 $\mathrm{V}$ obtained by voltammetry was linearly dependent on the meloxicam concentration in the range of 4.3 $\times 10^{-8} \sim 8.5 \times 10^{-6} \mathrm{M}$ in B-R $(0.04 \mathrm{M}, \mathrm{pH} 1.86)$ with a correlation coefficient of 0.999 and the 
detection limit was $1.5 \times 10^{-9} \mathrm{M}$. The electrochemical reaction mechanism of meloxicam could be concluded that the oxidation process of a meloxicam molecule include one $\mathrm{H}^{+}$and two single electrons. The modified electrode has also been demonstrated to be applicable in the detection of meloxicam in pharmaceutical formulation and spiked serum samples with excellent sensitivity and selectivity. This novel modifier is considered to be a promising, low-cost, and steady material for the modification of electrodes. The cysteic acid modified electrode will hopefully be of a good application for further sensor development.

\section{Acknowledgements}

This work was supported by the National Natural Science Foundation of China (No. 20375034) and the Novel Foundation of Jiangsu Provincial Education Department for Doctors (2005).

\section{References}

1. Noble, S. J.; Balfour, A. Meloxicam. Drugs 1996, 51, 424-430.

2. Turck, D.; Roth, W.; Busch, U. A review of the clinical pharmacokinetics of meloxicam. British Journal of Rheumatology 1996, 35, 13-16.

3. Engelhardt, G.; Homma, D.; Schlegel, K.; Utzmann, R.; Schnitzler, C. Anti-inflammatory, analgesic, antipyretic and related properties of meloxicam, a new non-steroidal anti-inflammatory agent with favorable gastrointestinal tolerance. Inflammation Research 1995, 44, 423-433.

4. Davies, N. M.; Skjodt, N. M. Clinical pharmacokinetics of meloxicam. A cyclo-oxygenase-2 preferential nonsteroidal anti-inflammatory drug. Clinical Pharmacokinetics 1999, 36, 115-126.

5. Martindale, The Extra Pharmacopoeia, Thirty second edition, The Pharmaceutical Press, London, England, 1999.

6. Hassan, E. M. Spectrophotometric and fluorimetric methods for the determination of meloxicam in dosage forms. Journal of Pharmaceutical and Biomedical Analysis 2002, 27, 771-777.

7. Garcia, Ma. S.; Sanchez-Pedreno, C. Ma.; Albero, I.; Marti, J. Spectrophotometric methods for determining meloxicam in pharmaceuticals using batch and flow-injection procedures. European Journal of Pharmaceutical Sciences 2000, 9, 311-316.

8. Joseph-Charles, J.; Bertucal, M. Determination of meloxicam in tablet formulations by ultraviolet spectrophotometry and high-performance liquid chromatography. Analytical Letters 1999, 32, 2051-2059.

9. Joseph-Charles, J.; Bertucal, M. Simultaneous high-performance liquid chromatography analysis of non-steroidal anti-inflammatory oxicams in pharmaceutical preparations. Journal of Liquid Chromatography Related Technologies 1999, 22, 2009-2021.

10. Meloxicam, The Chinese Pharmacopoeia, Chemical Industry Press, Beijing, China, 2005.

11. Emirhan, N.; Sedef, K. Method development and validation for the analysis of meloxicam in tablets by CZE. Joural of Pharmaceutical and Biomedical Analysis 2003, 31, 393-400.

12. Baeyens, W. R. G.; Vander Weken, G.; D’haeninck, E.; García-Campan, A. M.; Vankeirsbilck, T.; Vercauteren, A.; Deprez, P. Application of an alkyl-diol silica precolumn in a columns witching system for the determination of meloxicam in plasma. Journal of Pharmaceutical and Biomedical Analysis 2003, 32, 839-846. 
13. Beltagi, A. M.; Ghoneim, M. M.; Radi, A. Electrochemical reduction of meloxicam at mercury electrode and its determination in tablets. Journal of Pharmaceutical and Biomedical Analysis 2000, 28, 1501-1503.

14. Huang, H.; Gao, H. Y.; Zeng, Y. H. Single sweep oscillopolarography of meloxicam. Chinese Journal of Analytical Chemistry 2000, 17, 135-136.

15. Radi, A.; El Ries, M. A.; El-Anwar, F.; El-Sherif, Z. Electrochemical oxidation of meloxicam and its determination in tablet dosage form. Analytical Letters 2001, 34, 739-748.

16. Li, H.; Li, T.; Wang, E. Electrocatalytic oxidation and flow detection of cysteine at an aquocobalamin absorbed glassy carbon electrode. Talanta 1995, 42, 885-890.

17. Spataru, N.; Sarada, B. V.; Popa, E.; Tryk, D. A.; Fujishima, A. Voltammetric determination of $L$ cysteine at conductive diamond electrodes. Analytical Chemistry 2001, 73, 514-519.

18. Zagal, J.; Fierro, C.; Rozas, R. Electrocatalytic effects of adsorbed cobalt phthalocyanine tetrasulfonate in the anodic oxidation of cysteine. Journal of Electroanalytical Chemistry 1981, 119, 403-408.

19. Ralph, T. R.; Hitchman, M. L.; Millington, J. P.; Walsh, F. C. The electrochemistry of L-cystine and L-cysteine Part 1: Thermodynamic and kinetic studies. Journal of Electroanalytical Chemistry 1994, 375, 1-15.

20. Fei, S. D.; Cheng, J. H.; Yao, S. Z.; Deng, G. H.; He, D. L.; Kuang, Y. F. Electrochemical behavior of $L$-cysteine and its detection at carbon nanotube electrode modified with platinum. Analytical Biochemistry 2005, 339, 29-35.

21. Davis, D. G.; Bianco, E. An electrochemical study of the oxidation of $L$-cysteine. Journal of Electroanalytical Chemistry 1966, 12, 254-260.

22. Laviron, E. General expression of the linear potential sweep voltammogram in the case of diffusionless electrochemical systems. Journal of Electroanalytical Chemistry 1979, 101, 19-28.

(c) 2006 by MDPI (http://www.mdpi.org). Reproduction is permitted for noncommercial purposes. 